\title{
On the Estimation of Total Arterial Compliance from Aortic Pulse Wave Velocity
}

\author{
Orestis Vardoulis, Theodore G. Papaioannou, and Nikolaos Stergiopulos \\ Laboratory of Hemodynamics and Cardiovascular Technology, Institute of Bioengineering, Swiss Federal Institute of \\ Technology, Ecole Polytechnique Fédérale de Lausanne (EPFL)/STI/LHTC, BM 5128, Station 17, 1015 Lausanne, Switzerland
}

(Received 22 February 2012; accepted 19 May 2012; published online 31 May 2012)

Associate Editor Joan Greve oversaw the review of this article.

\begin{abstract}
Total arterial compliance $\left(C_{\mathrm{T}}\right)$ is a main determinant of cardiac afterload, left ventricular function and arterioventricular coupling. $C_{\mathrm{T}}$ is physiologically more relevant than regional aortic stiffness. However, direct, in vivo, non-invasive, measurement of $C_{\mathrm{T}}$ is not feasible. Several methods for indirect $C_{\mathrm{T}}$ estimation require simultaneous recording of aortic flow and pressure waves, limiting $C_{\mathrm{T}}$ assessment in clinical practice. In contrast, aortic pulse wave velocity (aPWV) measurement, which is considered as the "gold standard" method to assess arterial stiffness, is noninvasive and relatively easy. Our aim was to establish the relation between aPWV and $C_{\mathrm{T}}$. In total, 1000 different hemodynamic cases were simulated, by altering heart rate, compliance, resistance and geometry using an accurate, distributed, nonlinear, one-dimensional model of the arterial tree. Based on Bramwell-Hill theory, the formula $C_{\mathrm{T}}=k \cdot \mathrm{aPWV}^{-2}$ was found to accurately estimate $C_{\mathrm{T}}$ from aPWV. Coefficient $k$ was determined both analytically and by fitting $C_{\mathrm{T}}$ vs. aPWV data. $C_{\mathrm{T}}$ estimation may provide an additional tool for cardiovascular risk $(\mathrm{CV})$ assessment and better management of $\mathrm{CV}$ diseases. $C_{\mathrm{T}}$ could have greater impact in assessing elderly population or subjects with elevated arterial stiffness, where aPWV seem to have limited prognostic value. Further clinical studies should be performed to validate the formula in vivo.
\end{abstract}

Keywords-Arterial stiffness, PWV, Distensibility, Pressure waves, 1-D model, Elasticity, Windkessel, Wave speed, Systemic circulation.

\section{ABBREVIATIONS \\ $C_{\mathrm{T}} \quad$ Total arterial compliance \\ LV Left ventricle}

Address correspondence to Theodore G. Papaioannou, Laboratory of Hemodynamics and Cardiovascular Technology, Institute of Bioengineering, Swiss Federal Institute of Technology, Ecole Polytechnique Fédérale de Lausanne (EPFL)/STI/LHTC, BM 5128, Station 17, 1015 Lausanne, Switzerland. Electronic mail: teogpap@ gmail.com, thepap@med.uoa.gr work.

O. Vardoulis and T. G. Papaioannou contributed equally to this

$\begin{array}{ll}\text { PWV } & \text { Pulse wave velocity } \\ \text { aPWV } & \text { Aortic pulse wave velocity } \\ \text { CV } & \text { Cardiovascular } \\ \text { RMSE } & \text { Root mean square error } \\ \text { SSE } & \text { Sum of square errors } \\ \text { SD } & \text { Standard deviation } \\ \text { CI } & \text { Confidence intervals } \\ \text { BP } & \text { Blood pressure }\end{array}$

\section{INTRODUCTION}

Total arterial compliance $\left(C_{\mathrm{T}}\right)$ is a global biomechanical property of the arterial tree with major physiological $^{4,6}$ and pathological ${ }^{7,8,27}$ relevance. Compliance expresses the ability of the arterial system to store blood during systole without excessive pressure rise. $C_{\mathrm{T}}$ is an important determinant of central blood pressure and cardiac afterload. Additionally, it is a part of systemic vascular input impedance and affects cardiac energy requirements. A decrease in $C_{\mathrm{T}}$ can inhibit pressure and flow wave damping and increase left ventricular (LV) load, thus influencing LV function. $^{20,22}$ The reduction of $C_{\mathrm{T}}$ leads to an increase in wave speed, and consequently arrival of reflected waves during early systole, augmenting peak systolic pressure and pulse pressure. In parallel, coronary perfusion can be compromised due to the lower diastolic pressure.

Direct in vivo, non-invasive, measurement of $C_{\mathrm{T}}$ is not feasible. To overcome this limitation, several methods have been proposed for indirect estimation of $C_{\mathrm{T}}{ }^{13,30-33}$ Most commonly, these methods require simultaneous recording of the proximal aortic pressure wave and flow or cardiac output. Still, the complexity of these methods has limited the assessment of $C_{\mathrm{T}}$ in every day clinical practice, while other surrogates of local or regional arterial stiffness, ${ }^{14,29}$ have been used more often. 
Measurement of pulse wave velocity (PWV) is currently considered as the "gold standard" method to assess arterial stiffness. ${ }^{12}$ PWV can be utilized for the estimation of both local ${ }^{2,24}$ and regional arterial distensibility $^{12}$; the assessment of the latter is based on (i) the estimation of the pressure wave transit time between two arterial sites and (ii) the measurement of the distance between these two sites. There is emerging evidence supporting that particularly aortic PWV (aPWV)-i.e., between the carotid and femoral artery - is an independent predictor of $\mathrm{CV}$ risk and mortality ${ }^{29,36}$ and it is now widely used in clinical research. Nonetheless, there is no doubt that $C_{\mathrm{T}}$ is physiologically more relevant than regional or local arterial compliance (or their surrogate PWV), in terms of modulation of cardiac load, LV function, arterio-ventricular coupling and CV risk. Therefore, there is a need for understanding the nature and form of the relation between regional aortic PWV and total arterial compliance and, if possible, provide an analytical expression for deriving total arterial compliance from measured aPWV. The aim of the present study was to: (i) to examine how aPWV is associated with total systemic compliance and (ii) to provide a relationship for the estimation of $C_{\mathrm{T}}$ from aPWV. Accurate values of $C_{\mathrm{T}}$ are difficult to obtain in the intact organism. Subsequently, we designed an in silico approach using an accurate, physiological relevant, and validated one-dimensional model of the systemic arterial tree, where arterial properties were changed within their physiological ranges and precise readouts of aortic PWV and total arterial compliance were made to derive their corresponding relationship.

\section{MATERIALS AND METHODS}

\section{Estimation of Total Arterial Compliance}

A theoretical approach based on Bramwell-Hill theory ${ }^{3}$ was utilized in order to derive a mathematical equation relating directly $C_{\mathrm{T}}$ with aPWV. For each segment $i$ of the arterial tree the volumetric compliance $C_{i}$ is equal to:

$$
C_{i}=\frac{V_{i}}{\rho} \cdot \frac{1}{\mathrm{PWV}_{i}^{2}},
$$

where $\rho$ is blood density, $V_{i}$ is the volume and $\mathrm{PWV}_{i}$ is the wave speed in the segment $i$, respectively. The total volumetric arterial compliance $C_{\mathrm{T}}$ is the sum of the segments' volumetric compliance $\left(C_{i}\right)$ :

$$
C_{\mathrm{T}}=\sum_{i}^{n} C_{i}=\sum_{i}^{n}\left(\frac{V_{i}}{\rho} \cdot \frac{1}{\mathrm{PWV}_{i}^{2}}\right),
$$

where $n$ is the total number of arterial segments of the arterial tree.
We introduce aPWV in Eq. (2) by multiplying and dividing each term by $\mathrm{aPWV}^{2}$ :

$$
C_{\mathrm{T}}=\sum_{i}^{n}\left(\frac{V_{i}}{\rho} \cdot \frac{\mathrm{aPWV}^{2}}{\mathrm{PWV}_{i}^{2}}\right) \cdot \frac{1}{\mathrm{aPWV}^{2}}
$$

The term

$$
\sum_{i}^{n}\left(\frac{V_{i}}{\rho} \cdot \frac{\mathrm{aPWV}^{2}}{\mathrm{PWV}_{i}^{2}}\right)=k
$$

is a coefficient $k$ accounting for the contribution of local geometry and wave speed of each arterial segment and allowing to link with a simple power relation $C_{\mathrm{T}}$ and aPWV:

$$
C_{\mathrm{T}}=k \cdot \frac{1}{\mathrm{aPWV}^{2}}
$$

Since PWV and arterial volume cannot be practically measured for each arterial segment in vivo, the term $k$ is determined in silico using a validated one-dimensional model of the systemic arterial tree, accounting for all major arterial branches. The model was previously described and validated in vivo. ${ }^{25,26} \mathrm{We}$ simulated 1000 different hemodynamic cases by modifying arterial compliance, resistance, heart rate and the geometry of the arterial segments. In the arterial tree model of Reymond ${ }^{26}$ the compliance is non-linearly related to pressure. As described by Langewouters ${ }^{10}$ the relationship requires a reference value of compliance (in this case at $100 \mathrm{mmHg}$ ) that is then multiplied with a non-dimensional, pressure dependent factor in order to produce the compliance at each pressure level. In the current study, the reference compliance of all segments in the arterial tree and the resistance of the terminal arterial sites were changed uniformly according to randomly selected scaling factors (range: 0.1-1.0 and 0.6-2.0 for the reference compliance and the resistance, respectively). The aforementioned changes in arterial compliance and resistance were performed for 3 different heart rates $(75,80,85 \mathrm{bpm})$ and for three different geometric configurations of the arterial tree that were generated by reducing or increasing the of the arterial segments (thereby volumes) by -10 and $+10 \%$, respectively. As a result, a variety of different hemodynamic and vascular mechanical properties were simulated (Table 1), providing also various pressure wave patterns along the arterial tree. The governing equations of the model are obtained by integrating the continuity and longitudinal momentum equations (Navier-Stokes) over the arterial crosssection to yield their 1-D form. The arterial behavior was considered to be viscoelastic and nonlinear, using the methodology of Holenstein et al. ${ }^{9}$ based on the published data of Bergel. ${ }^{1}$ Each segment of the arterial 
TABLE 1. Descriptive characteristics of the 1000 simulated cases.

\begin{tabular}{|c|c|c|c|c|}
\hline & Min & Max & Mean & SD \\
\hline Aortic systolic BP (mmHg) & 81.4 & 179.2 & 131.0 & 18.66 \\
\hline Aortic diastolic BP (mmHg) & 40.4 & 123.4 & 78.7 & 18.69 \\
\hline Aortic pulse pressure $(\mathrm{mmHg})$ & 22.4 & 128.7 & 52.3 & 22.62 \\
\hline Brachial systolic BP $(\mathrm{mmHg})$ & 87.9 & 179.6 & 142.6 & 17.90 \\
\hline Brachial diastolic BP $(\mathrm{mmHg})$ & 37.8 & 121.6 & 76.2 & 18.61 \\
\hline Brachial pulse pressure $(\mathrm{mmHg})$ & 30.6 & 131.5 & 66.5 & 22.39 \\
\hline Mean arterial BP (mmHg) & 65.6 & 138.3 & 104.8 & 15.59 \\
\hline Total arterial compliance $(\mathrm{mL} / \mathrm{mmHg})$ & 0.13 & 2.26 & 0.80 & 0.39 \\
\hline Total vascular resistance $(\mathrm{mmHg} \mathrm{s} / \mathrm{mL})$ & 0.60 & 1.68 & 1.16 & 0.27 \\
\hline Estimated carotid-to-femoral PWV (m/s) & 4.07 & 11.76 & 7.08 & 1.37 \\
\hline
\end{tabular}

BP: blood pressure; PWV: pulse wave velocity; SD: standard deviation.

model was considered as long tapered tube and compliance was defined by a non-linear function of pressure based on the equation proposed by Langerwouters. ${ }^{10}$ LV function was simulated by the varying elastance model described by Sagawa. ${ }^{28}$ Distal vessels were terminated with three-element Windkessel models. Intimal shear was modeled using the Witzig-Womersley theory. Pressure and flow throughout the systemic tree were obtained by solving the governing equations of the model with proper boundary conditions, using an implicit finite difference scheme.

\section{Calculation of Pulse Wave Velocity and Total Arterial Compliance}

Aortic pulse wave velocity (aPWV) was estimated by determining the transit time via pulse wave analysis, similarly to the in vivo methodologies for aPWV measurement. ${ }^{12}$ The transit time between proximal and distal pressure waveforms was determined with an automated technique that located the minimum diastolic pressure in the waveform. ${ }^{5}$ The waveforms were recorded at arterial sites that resemble aPWV measurement in clinical practice; namely, the carotid and femoral artery (carotid-to-femoral PWV), as previously described, ${ }^{12,34}$ and shown schematically in Fig. 1 (Sites A and B, respectively). The "real" $C_{\mathrm{T}}$ for each of the 1000 simulated cases, was directly calculated as the summation of volumetric compliance for all the arterial segments (at mean pressure), including the compliance of the terminal sites (Windkessel models). The resulting values of total compliance ranged between 0.126 and $2.256 \mathrm{~mL} / \mathrm{mmHg}$. Peripheral resistance had a minimum of $0.600 \mathrm{mmHg} \mathrm{s} / \mathrm{mL}$ and a maximum of $1.681 \mathrm{mmHg} \mathrm{s} / \mathrm{mL}$. The computed $C_{\mathrm{T}}$ and aPWV values were fitted according to the nonlinear power relationship given by Eq. (5). The following fit quality parameters were calculated: sum of square errors (SSE) $R^{2}$, and root mean square error (RMSE).
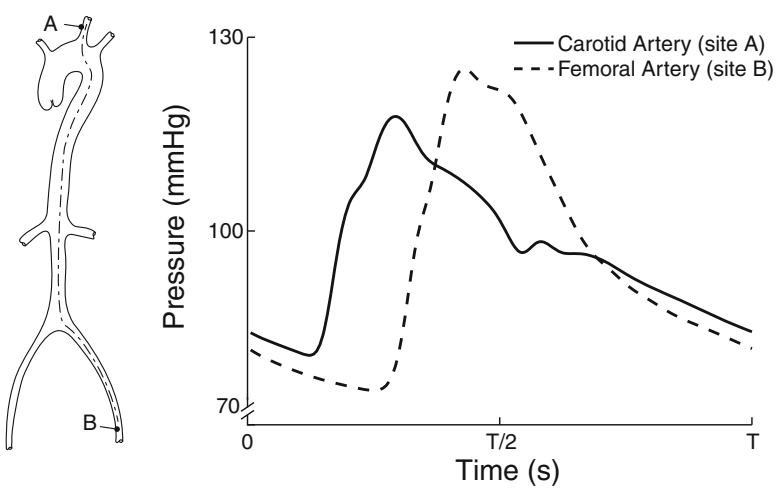

FIGURE 1. Measurement of aPWV by estimating "transit time", of pressure waves between: carotid (A) and femoral (B) artery.

\section{RESULTS}

The descriptive values of hemodynamic and vascular parameters derived from the 1000 cases are reported in Table 1.

The value of $k$, derived by fitting aPWV and $C_{\mathrm{T}}$ values, was 36.7 (95\% CI: 36.2-37.2). Goodness of fit parameters were also calculated; the SSE was 29.94, the $R^{2}$ was 0.8 and the RMSE was 0.17 . The value of $k$ was also determined analytically according to Eq. (4) and it was found to be 39.4.

The following formula, which is a generalized form of the Bramwell-Hill equation, is proposed for the prediction of total systemic compliance from regional aPWV:

$$
C_{\mathrm{T}}=37 \cdot \frac{1}{\mathrm{aPWV}^{2}},
$$

where $C_{\mathrm{T}}$ is given in $\mathrm{mL} / \mathrm{mmHg}$ and aPWV in $\mathrm{m} / \mathrm{s}$. aPWV is the estimated carotid-to-femoral PWV, which corresponds to the commonly used methodology for the assessment of aortic stiffness in clinical practice.

The fit curve for $C_{\mathrm{T}}$ and aPWV values computed for the 1000 cases is illustrated in Fig. 2. Figure 3 shows the correlation between the total compliance and the 
predicted values of compliance via the fitted curve. The hemodynamic cases were divided into three subgroups based on the geometric change that was imposed in the arterial trees. The fit curves were also calculated for each of the three "arterial-size" subgroups and the $k$ values were $32.8,36.9$ and 41.2 for the $10 \%$ decrease, control and $+10 \%$ increase in arterial volume, respectively. Table 2 summarizes the fit results for all the curves.

\section{DISCUSSION}

A new relationship was established between total arterial compliance and regional aPWV. To our

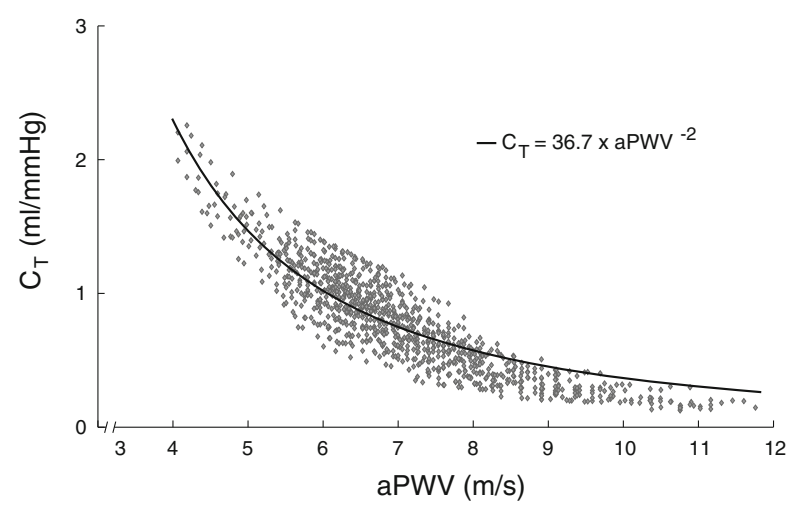

FIGURE 2. Power relationship between total arterial compliance $\left(C_{\mathrm{T}}\right)$ and aPWV.

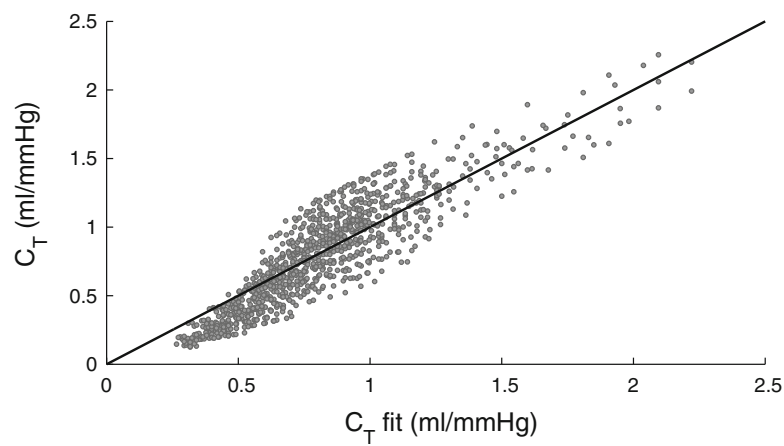

FIGURE 3. Scatter plot between the values of total arterial compliance derived from the model and the values of total arterial compliance calculated by the fitted curve (solid line represents equality). knowledge, this is the first study providing an explicit relationship between total arterial compliance and aortic (carotid-to-femoral) PWV. The formula is based on a generalized form of the Bramwell-Hill equation for local PWV and appears to apply well over a wide range of simulated physiological conditions.

\section{Nature of the Generalized Bramwell-Hill Law and Physiological Significance of Its Parameters}

Senso stricto, the Bramwell-Hill equation is applied to derive local $\mathrm{PWV}$ from local area compliance $\left(\mathrm{PWV}=\left(A / \rho^{*} 1 / C_{\mathrm{A}}\right)^{1 / 2}\right)$. Neglecting tapering and multiplying local cross-sectional area, $A$, and local area compliance, $C_{\mathrm{A}}$, by the length of the arterial segment under consideration, we may express segment volume compliance as a function of average PWV in the same segment. Summing up the compliance in all arterial segments we derive the generalized Bramwell-Hill law expressed in Eq. (3). We went further to express this generalized Bramwell-Hill equation in terms of a clinically relevant aortic PWV, such as the carotidto-femoral PWV. This gave rise to a generalized Bramwell-Hill equation with a single coefficient $k$, whose analytical form is given in Eq. (4). The coefficient $k$ is principally dependent on geometry and, in specific, on the weighted sum of all arterial segment volumes. The weighting coefficient of each segment volume is $1 / \rho *\left(\mathrm{aPWV}^{2} / \mathrm{PWV}_{i}^{2}\right)$, with the ratio

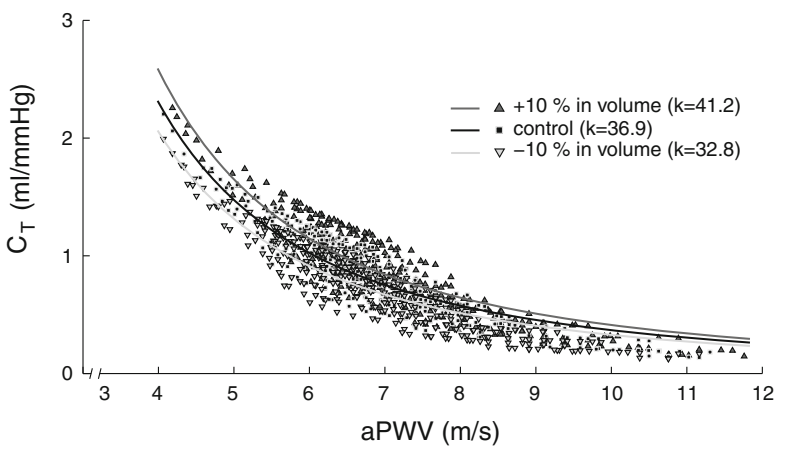

FIGURE 4. Power relationship between total arterial compliance $\left(C_{\mathrm{T}}\right)$ and aPWV for the three subgroups of different arterial size $(-10 \%$, control and $+10 \%)$.

TABLE 2. Results for the curve parameters and the quality of fit.

\begin{tabular}{lcccc}
\hline & $k$ & SSE & $R^{2}$ & RMSE \\
\hline Total $(N=1000)$ & 36.7 & 29.9 & 0.800 & 0.173 \\
$+10 \%$ in arterial volume $(N=326)$ & 41.2 & 9.29 & 0.832 & 0.169 \\
Control $(N=331)$ & 36.9 & 7.32 & 0.848 & 0.149 \\
$-10 \%$ in arterial volume $(N=343)$ & 32.8 & 6.73 & 0.846 & 0.140 \\
\hline
\end{tabular}

SSE: sum of square errors; RMSE: root mean square error.

Control refers to the reference arterial tree model as described by Reymond et a ${ }^{26}$. 
aPWV/PWV ${ }_{i}$ being quite comparable between individuals. Figure 4 shows the fit curves that were calculated for three subgroups based on the geometry change that was performed. There was a very good agreement regarding the proportionality of the $k$ values to the volume of the arterial tree. The $10 \%$ increase of the arterial cross-sectional areas (and subsequently volume) resulted in an $11.8 \%$ increase in the value of $k$, whereas $10 \%$ volume decrease resulted in a $10.8 \%$ decrease for the power relationship constant. Hence, overall, we expect coefficient $k$ to be principally body size dependent, but this needs to be verified with further studies either in silico or with in vivo measurements.

\section{Relevance of Total Arterial Compliance}

Total arterial compliance and total arterial resistance constitute the two main parameters defining the global biomechanical properties of the arterial system. Total arterial compliance is a major determinant of cardiac load, LV function, arterio-ventricular coupling and eventually $\mathrm{CV}$ risk. Modeling vasculature and hemodynamic responses often require estimation of $C_{\mathrm{T}}$, while other methods for minimally invasive cardiac output monitoring based on pulse contour analysis (PCCO) are also dependent on $C_{\mathrm{T}}$ values. Total arterial compliance is, however, a parameter difficult to estimate in clinical practice, as it ideally needs measurement of pressure and flow in the proximal aorta. ${ }^{32,33}$ The proposed relationship for the estimation of $C_{\mathrm{T}}$, from aPWV alone, may provide a simple way for in vivo assessment of total arterial compliance. Knowledge of $C_{\mathrm{T}}$ might have additional diagnostic impact as well as additive prognostic value beyond PWV. In contrast to PWV, which can be measured directly and noninvasively, existing methods for $C_{\mathrm{T}}$ estimation have not entered the everyday clinical or research practice. This was mainly due to inherent limitations, such as methodological complexity and lack of validation. Consequently, up to now, there are no adequate clinical data for the prognostic value of $C_{\mathrm{T}}{ }^{37}$ For this reason, the use of aPWV for the estimation of $C_{\mathrm{T}}$, may provide a means for assessing total systemic compliance indirectly in large populations and this is the main advantage of the proposed equation.

\section{PWV as Indicator of Arterial Stiffness}

Total arterial compliance, the inverse of arterial elastance or stiffness, characterizes the entire arterial tree. PWV, in accordance to Moens-Kortewg's relationship, is proportional to the square root of local elastic modulus, thereby characterizing the local stiffness at a given arterial location. When PWV is calculated from the wave travel time over a given arterial length, it characterizes the average stiffness of the arterial segments included in the traveled path. When the arterial path includes the aorta, and especially the ascending and thoracic aorta where more than $50 \%$ of total arterial compliance resides, it is logical to assume that the measured PWV will reflect largely the global stiffness of the arterial tree. Our results clearly support such hypothesis, as seen in the relationship between $C_{\mathrm{T}}$ and aPWV shown in Fig. 2. The relationship is well approximated by a generalized Bramwell-Hill type of law, further supporting the inverse relationship between total arterial compliance and the square of aPWV. In that respect, our findings do support the well accepted postulate that aPWV can be used as indicator of total arterial stiffness and not only strictly local arterial stiffness. There is plenty of evidence indicating that arterial stiffness (assessed by PWV) is associated with the presence of $\mathrm{CV}$ risk factors and atherosclerotic disease. ${ }^{17}$ More importantly, PWV is an independent predictor of CV mortality in endstage renal disease, ${ }^{21}$ hypertension ${ }^{11}$ and in general population. $^{19}$

The existing clinical data and the simplicity of pulse wave speed measurement led to the inclusion of PWV in guidelines for the management of arterial hypertension $^{15}$; the European Society of Hypertension/European Society of Cardiology suggests PWV as a tool for assessment of subclinical target organ damage. However, although there is ample evidence supporting that PWV can predict CV risk and mortality, ${ }^{36}$ in several conditions PWV has limited predictive value ${ }^{18,23,35}$ which could be related to the sensitivity of PWV to "predict" total arterial compliance. It has been shown that in a population of 331 elderly patients $>70$ years, with increased arterial stiffness and high PWV, the aPWV did not predict CV mortality. ${ }^{23}$ In another study in 205 patients (mean age 65 years) who underwent coronary angiography, brachial-ankle PWV was not an independent predictor of coronary artery disease severity. ${ }^{16}$ Again in this study PWV was high $(16.4 \pm 3.6 \mathrm{~m} / \mathrm{s})$. A previous study performed within the framework of the Rotterdam Study, investigated whether aortic stiffness (assessed by PWV) improves the prediction of coronary heart disease in 2,849 elderly subjects with mean age 71.5 years. ${ }^{35}$ It was observed that there is low additional value of aortic stiffness in the clinical management of coronary heart disease in elderly patients with $\mathrm{CV}$ disease and high arterial stiffness (mean PWV $13.3 \mathrm{~m} / \mathrm{s}$ ). ${ }^{35}$ In another clinical study, aPWV and distensibility did not predict coronary and extracoronary atherosclerosis in asymptomatic men at risk for $\mathrm{CV}$ disease. ${ }^{18}$ The aforementioned studies seem to suggest a limitation of PWV to predict CV risk and mortality in the elderly or in subjects with increased arterial stiffness. A possible 


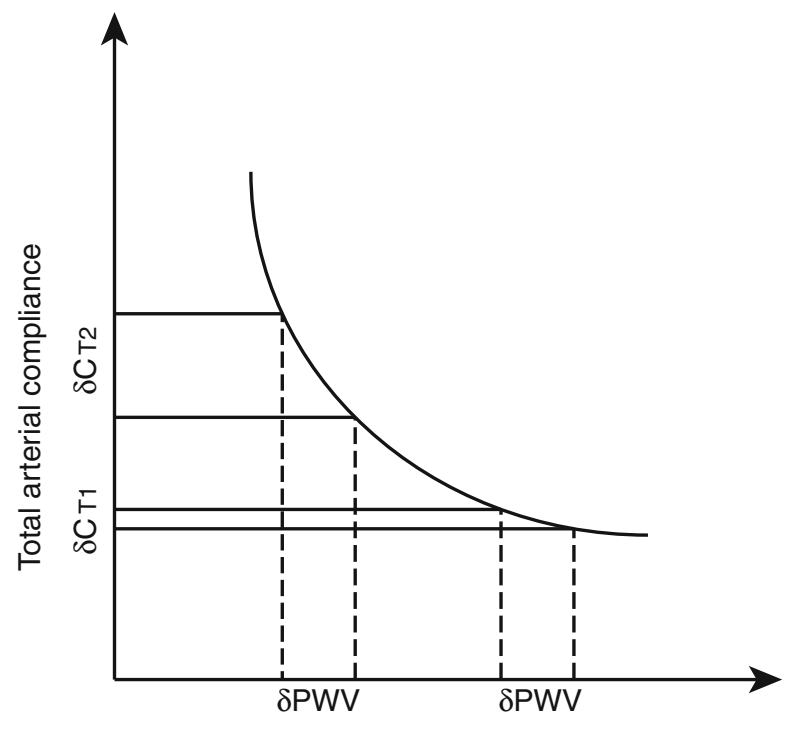

Aortic pulse wave velocity

FIGURE 5. Relative effects of aPWV changes on total arterial compliance based on their nonlinear relationship.

explanation for this maybe the fact that $C_{\mathrm{T}}$ is related with aPWV through an inverse non-linear curve as illustrated schematically in Fig. 5.

A specific change in PWV $\left(\delta_{\mathrm{PWV}}\right)$ at higher levels of arterial stiffness (higher PWV) is related to small changes in total compliance $\delta C_{\mathrm{T} 1}$ and thus it may have a small incremental impact on cardiac afterload and function. In contrast, the same change $\delta_{\mathrm{PWV}}$ at lower levels of PWV is related to a substantial change $\delta C_{\mathrm{T} 2}$ of total compliance (Fig. 5), and thus may influence cardiac load and performance to a greater extent. Hence, the sensitivity of PWV to reflect $C_{\mathrm{T}}$ at high levels of arterial stiffness (often observed in the elderly) is much less when compared to lower levels of PWV (lower arterial stiffness). Since $C_{\mathrm{T}}$ is physiologically more relevant compared to regional or local PWV, the proposed relation could be utilized for $\mathrm{CV}$ risk assessment in elderly populations or in patients with increased levels of arterial stiffness. The estimation of $C_{\mathrm{T}}$ can provide an additional tool for better prediction and management of $\mathrm{CV}$ risk, as well as for studying pathophysiological or pharmaceutical mechanisms related to total arterial compliance changes.

\section{Methodological Issues, Limitations and Future Perspectives}

It should be acknowledged that the proposed relationship between aPWV and $C_{\mathrm{T}}$, was based on both basic physical principles and in silico data derived by a one-dimensional model of the arterial tree. This model has been previously validated in vivo ${ }^{25,26}$ and provides realistic pressure and flow waves. Thus, it is able to mimic the real hemodynamic profiles within a wide range of $C_{\mathrm{T}}$ and PWV values. Additionally, this model allows the direct accurate calculation of $C_{\mathrm{T}}$ in several different hemodynamic conditions, which is not possible to be directly performed in vivo by non-invasive means. Obviously, the ultimate validation of Eq. (5) can only come from large scale in vivo data covering different gender and age groups as well as different physiological conditions and disease states, where aPWV and $C_{\mathrm{T}}$ are carefully measured with appropriate and mutually independent methods, such as transit time for PWV and pulse pressure method or equivalent for $C_{\mathrm{T}}$. It should be also noted that in our simulations, we did not perform changes in mechanical or geometrical properties of specific arterial sites, but we studied global changes. This might have an impact in the derived total compliance by the proposed formula especially at higher levels of PWV.

A few issues should be addressed regarding the physiological relevance of $k$ coefficient. Based on Eq. (4), $k$ is proportional to total arterial volume $\left(k \sim V_{\mathrm{T}} / \rho\right)$, assuming that the ratios $\mathrm{aPWV}^{2} / \mathrm{PWV}_{i}^{2}$ are relatively constant among different cases/subjects. Since $V_{\mathrm{T}}$ cannot be measured (in vivo) directly, it can be assumed that it is proportional to the product of a characteristic arterial cross sectional area and a characteristic arterial length, namely $V_{\mathrm{T}} \sim A \times L$. If we further assume that cross-sectional areas of arterial segments are proportional to the cross-sectional area of the aorta $\left(A_{\mathrm{a}}\right)$ and segment lengths are proportional to height $(h), k$ can be expressed as: $k=k^{\prime} \times A_{\mathrm{a}} \times$ $h / \rho$. This would lead to the following empirical relationship:

$$
C_{\mathrm{T}}=k^{\prime} \frac{A_{\mathrm{a}} h}{\rho} \frac{1}{\mathrm{aPWV}^{2}},
$$

where $k^{\prime}$ is now a non-dimensional coefficient, presumably independent of body size. The above alternate formula needs to be tested on a large number of subjects with different body sizes in order to assess the validity of the underlying hypotheses and verify that coefficient $k^{\prime}$ is indeed independent of body size.

The $k$ value of the fit was $6.9 \%$ lower than the $k$ value derived analytically (Eq. (4)). This difference can be attributed: (i) to the fact that foot-to-foot methods produce PWV results that correspond to diastolic pressure and thus higher values of compliance ${ }^{10}$ and (ii) to the fact that Eq. (4) was derived by the Bramwell-Hill equation that does not take into account viscoelastic properties of the arterial walls, in contrast to our 1-D model.

The advantage of the proposed equation for $C_{\mathrm{T}}$ estimation from aPWV compared to other methods based on aortic pressure and flow wave analysis is mostly methodological and practical. Aortic velocity 
profiles can be recorded by ultrasound Doppler or MRI, but these measurements cannot be widely performed in every day clinical routine. Furthermore, the recording of aortic pressure waves require either the use of transfer functions in order to derive aortic from peripheral (i.e., radial or brachial) pressure waves or the use of catheter pressure transducers. Both approaches are often limited due to inaccuracies or invasiveness, respectively. Moreover, some methods (such as the "Decay time" or the "Pulse pressure method") process pressure and flow waves in the Fourier domain thus requiring precise wave recordings. In the majority of research or clinical cases, whenever the value of total compliance is required, it is rather difficult to perform such measurements. On the other hand carotid-to-femoral PWV can be easily and non-invasively assessed by simple techniques that are widely used in clinical practice for the assessment of aortic stiffness. Thus aPWV can provide an easy, low cost and simple mean to determine total arterial compliance in a routine basis.

\section{CONCLUSIONS}

It was demonstrated that aPWV reflects total arterial compliance following a non-linear power equation. The proposed relation allows the estimation of $C_{\mathrm{T}}$ only from aPWV. Since aPWV is a commonly and easily measured surrogate of arterial stiffness in clinical practice, $C_{\mathrm{T}}$ could be easily assessed, providing an additional research and clinical tool for $\mathrm{CV}$ risk assessment and better management of $\mathrm{CV}$ diseases. The proposed formula could possibly have greater impact when applied in elderly populations or subjects with elevated arterial stiffness, where PWV seems to have limited prognostic value. Further clinical studies remain to be performed in order to verify this hypothesis and also to validate the new relationship between $C_{\mathrm{T}}$ and aPWV with in vivo data.

\section{CONFLICT OF INTEREST}

The authors have no conflict of interest or financial support to declare.

\section{REFERENCES}

\footnotetext{
${ }^{1}$ Bergel, D. H. The dynamic elastic properties of the arterial wall. J. Physiol. 156:458-469, 1961.

${ }^{2}$ Borlotti, A., S. Vermeersch, E. Rietzschel, P. Segers, and A. W. Khir. A comparison between local wave speed in the carotid and femoral arteries in healthy humans: application of a new method. Conf. Proc. IEEE Eng. Med. Biol. Soc. 2010:2857-2860, 2010.
}

${ }^{3}$ Bramwell, J. C., and A. V. Hill. The velocity of the pulse wave in man. Proc. R. Soc. Lond. B 93:298-306, 1922.

${ }^{4}$ Chemla, D., I. Antony, Y. Lecarpentier, and A. Nitenberg. Contribution of systemic vascular resistance and total arterial compliance to effective arterial elastance in humans. Am. J. Physiol. Heart Circ. Physiol. 285:H614 H620, 2003.

${ }^{5}$ Chiu, Y. C., P. W. Arand, S. G. Shroff, T. Feldman, and J. D. Carroll. Determination of pulse wave velocities with computerized algorithms. Am. Heart J. 121:1460-1470, 1991.

${ }^{6}$ de Simone, G., M. J. Roman, S. R. Daniels, G. Mureddu, T. R. Kimball, R. Greco, and R. B. Devereux. Age-related changes in total arterial capacitance from birth to maturity in a normotensive population. Hypertension 29:1213-1217, 1997.

${ }^{7}$ Haluska, B. A., L. Jeffriess, M. Downey, S. G. Carlier, and T. H. Marwick. Influence of cardiovascular risk factors on total arterial compliance. J. Am. Soc. Echocardiogr. 21:123-128, 2008.

${ }^{8}$ Haluska, B. A., K. Matthys, R. Fathi, E. Rozis, S. G. Carlier, and T. H. Marwick. Influence of arterial compliance on presence and extent of ischaemia during stress echocardiography. Heart 92:40-43, 2006.

${ }^{9}$ Holenstein, R., P. Niederer, and M. Anliker. A viscoelastic model for use in predicting arterial pulse waves. J. Biomech. Eng. 102:318-325, 1980.

${ }^{10}$ Langerwouters, G. J. Visco-elasticity of the human aorta in vitro in relation to pressure and age. Ph.D. Dissertation, Vrije Universiteit, Amsterdam, p. 221, 1982.

${ }^{11}$ Laurent, S., P. Boutouyrie, R. Asmar, I. Gautier, B. Laloux, L. Guize, P. Ducimetiere, and A. Benetos. Aortic stiffness is an independent predictor of all-cause and cardiovascular mortality in hypertensive patients. Hypertension 37:1236-1241, 2001.

${ }^{12}$ Laurent, S., J. Cockcroft, L. Van Bortel, P. Boutouyrie, C. Giannattasio, D. Hayoz, B. Pannier, C. Vlachopoulos, I. Wilkinson, and H. Struijker-Boudier. Expert consensus document on arterial stiffness: methodological issues and clinical applications. Eur. Heart J. 27:2588-2605, 2006.

${ }^{13}$ Liu, Z., K. P. Brin, and F. C. Yin. Estimation of total arterial compliance: an improved method and evaluation of current methods. Am. J. Physiol. 251:H588-H600, 1986.

${ }^{14}$ Mackenzie, I. S., I. B. Wilkinson, and J. R. Cockcroft. Assessment of arterial stiffness in clinical practice. QJM 95:67-74, 2002.

${ }^{15}$ Mancia, G., G. De Backer, A. Dominiczak, R. Cifkova, R. Fagard, G. Germano, G. Grassi, A. M. Heagerty, S. E. Kjeldsen, S. Laurent, et al. Guidelines for the management of arterial hypertension: the task force for the management of arterial hypertension of the European Society of Hypertension (ESH) and of the European Society of Cardiology (ESC). J. Hypertens. 25:1105-1187, 2007.

${ }^{16}$ Matsushima, Y., H. Kawano, Y. Koide, T. Baba, G. Toda, S. Seto, and K. Yano. Relationship of carotid intima-media thickness, pulse wave velocity, and ankle brachial index to the severity of coronary artery atherosclerosis. Clin. Cardiol. 27:629-634, 2004.

${ }^{17}$ Mattace-Raso, F. U., T. J. van der Cammen, A. Hofman, N. M. van Popele, M. L. Bos, M. A. Schalekamp, R. Asmar, R. S. Reneman, A. P. Hoeks, M. M. Breteler, et al. Arterial stiffness and risk of coronary heart disease and stroke: the Rotterdam Study. Circulation 113:657-663, 2006.

${ }^{18}$ Megnien, J. L., A. Simon, N. Denarie, M. Del-Pino, J. Gariepy, P. Segond, and J. Levenson. Aortic stiffening 
does not predict coronary and extracoronary atherosclerosis in asymptomatic men at risk for cardiovascular disease. Am. J. Hypertens. 11:293-301, 1998.

${ }^{19}$ Mitchell, G. F., S. J. Hwang, R. S. Vasan, M. G. Larson, M. J. Pencina, N. M. Hamburg, J. A. Vita, D. Levy, and E. J. Benjamin. Arterial stiffness and cardiovascular events: the Framingham Heart Study. Circulation 121:505-511, 2010.

${ }^{20}$ Mottram, P. M., B. A. Haluska, R. Leano, S. Carlier, C. Case, and T. H. Marwick. Relation of arterial stiffness to diastolic dysfunction in hypertensive heart disease. Heart 91:1551-1556, 2005.

${ }^{21}$ Pannier, B., A. P. Guerin, S. J. Marchais, M. E. Safar, and G. M. London. Stiffness of capacitive and conduit arteries: prognostic significance for end-stage renal disease patients. Hypertension 45:592-596, 2005.

${ }^{22}$ Papaioannou, T. G., D. S. Mathioulakis, and S. G. Tsangaris. Simulation of systolic and diastolic left ventricular dysfunction in a mock circulation: the effect of arterial compliance. J. Med. Eng. Technol. 27:85-89, 2003.

${ }^{23}$ Protogerou, A. D., M. E. Safar, P. Iaria, H. Safar, K. Le Dudal, J. Filipovsky, O. Henry, P. Ducimetiere, and J. Blacher. Diastolic blood pressure and mortality in the elderly with cardiovascular disease. Hypertension 50:172180, 2007.

${ }^{24}$ Rabben, S. I., N. Stergiopulos, L. R. Hellevik, O. A. Smiseth, S. Slordahl, S. Urheim, and B. Angelsen. An ultrasoundbased method for determining pulse wave velocity in superficial arteries. J. Biomech. 37:1615-1622, 2004.

${ }^{25}$ Reymond, P., Y. Bohraus, F. Perren, F. Lazeyras, and N. Stergiopulos. Validation of a patient-specific onedimensional model of the systemic arterial tree. Am. J. Physiol. Heart Circ. Physiol. 301:H1173-H1182, 2011.

${ }^{26}$ Reymond, P., F. Merenda, F. Perren, D. Rufenacht, and N. Stergiopulos. Validation of a one-dimensional model of the systemic arterial tree. Am. J. Physiol. Heart Circ. Physiol. 297:H208-H222, 2009.

${ }^{27}$ Safar, M. E., and G. M. London. Arterial and venous compliance in sustained essential hypertension. Hypertension 10:133-139, 1987.
${ }^{28}$ Sagawa, K. Cardiac Contraction and the Pressure-Volume Relationship. Oxford, UK: Oxford University Press, 1988.

${ }^{29}$ Sakuragi, S., and W. P. Abhayaratna. Arterial stiffness: methods of measurement, physiologic determinants and prediction of cardiovascular outcomes. Int. J. Cardiol. 138:112-118, 2010.

${ }^{30}$ Segers, P., P. Verdonck, Y. Deryck, S. Brimioulle, R. Naeije, S. Carlier, and N. Stergiopulos. Pulse pressure method and the area method for the estimation of total arterial compliance in dogs: sensitivity to wave reflection intensity. Ann. Biomed. Eng. 27:480-485, 1999.

${ }^{31}$ Stergiopulos, N., J. J. Meister, and N. Westerhof. Simple and accurate way for estimating total and segmental arterial compliance: the pulse pressure method. Ann. Biomed. Eng. 22:392-397, 1994.

${ }^{32}$ Stergiopulos, N., J. J. Meister, and N. Westerhof. Evaluation of methods for estimation of total arterial compliance. Am. J. Physiol. 268:H1540-H1548, 1995.

${ }^{33}$ Stergiopulos, N., P. Segers, and N. Westerhof. Use of pulse pressure method for estimating total arterial compliance in vivo. Am. J. Physiol. 276:H424-H428, 1999.

${ }^{34}$ Van Bortel, L. M., S. Laurent, P. Boutouyrie, P. Chowienczyk, J. K. Cruickshank, T. De Backer, J. Filipovsky, S. Huybrechts, F. U. Mattace-Raso, A. D. Protogerou, et al. Expert consensus document on the measurement of aortic stiffness in daily practice using carotid-femoral pulse wave velocity. J. Hypertens. 30:445-448, 2012.

${ }^{35}$ Verwoert, G. C., S. E. Elias-Smale, D. Rizopoulos, M. T. Koller, E. W. Steyerberg, A. Hofman, M. Kavousi, E. J. Sijbrands, A. P. Hoeks, R. S. Reneman, et al. Does aortic stiffness improve the prediction of coronary heart disease in elderly? The Rotterdam Study. J. Hum. Hypertens. 26:2834, 2012.

${ }^{36}$ Vlachopoulos, C., K. Aznaouridis, and C. Stefanadis. Prediction of cardiovascular events and all-cause mortality with arterial stiffness: a systematic review and meta-analysis. J. Am. Coll. Cardiol. 55:1318-1327, 2010.

${ }^{37}$ Zoungas, S., and R. P. Asmar. Arterial stiffness and cardiovascular outcome. Clin. Exp. Pharmacol. Physiol. 34:647-651, 2007. 